\section{The effect of varying percentage of positive and negative instances and instructions on conjunctive and disjunctive learning ${ }^{1}$}

NANCY WADSWORTH DENNEY, University of Washington, Seattle, Wash. 98105

Conjunctive and disjunctive problems were compared, using two different stimulus lists. The results indicated that both conjunctive and disjunctive problems were solved significantly faster when the percentages of positive and negative instances were equal rather than when the naturally occurring percentages of positive and negative instances were used. Two sets of instructions were also compared. Typical concept-learning instructions led to significantly better performance on both problems than did instructions in which $S$ was told that some of the patterns would be "yeses" and some would be "nos." The three-way interaction between Rule, Instructions, and Percentage of Positive and Negative Instances was also significant. This interaction was due to the lack of difference between the conjunctive and disjunctive problems in the unequal, yes-no condition. In all other conditions, the conjunctive problem was significantly easier than the disjunctive problem.

In experiments designed to compare the learning of conjunctive and disjunctive concepts, Es have used either naturally occurring (Conant \& Trabasso, 1964) or equalized (King, 1966) percentages of positive and negative instances to construct their stimulus lists. However, no comparison of the effects of these different types of stimulus lists on performance has ever been made. Accordingly, one aim of the present experiment was to investigate performance on conjunctive and disjunctive problems under each of these conditions to determine whether or not these two percentages of positive and negative instances have similar effects on conceptual behavior.

A second variable, instructions, was also investigated, since investigators working with different $\mathrm{S}$ populations have employed different sets of instructions when comparing conjunctive and disjunctive problems. For example, King (1966), studying rule learning in children, used a simplified set of instructions that differed from the typical concept-learning instructions given adults; he told his Ss that some of the stimuli would be "positive" and some would be "negative," rather than that some of the stimuli would be examples of "the concept." There is some evidence with children (Denney, 1969) that the type of instructions does influence the relative difficulty of learning conjunctive and disjunctive concepts; under instructions similar to King's, no difference was obtained between the two problems, while under instructions more similar to those employed in adult concept learning studies, the disjunctive problem was significantly more difficult. The present experiment, employing college students as Ss, had as its second aim a comparison of a set of simplified instructions similar to those used in both the King and Denney studies with a set of typical concept learning instructions to see if similar effects would be obtained with this class of Ss.

\section{SUBJECTS}

The Ss were 80 introductory psychology students from the University of Washington. Ss were assigned randomly, but in equal numbers, to eight experimental conditions; each served individually for one experimental session lasting approximately $15 \mathrm{~min}$.

\section{STIMULI}

Stimulus cards were geometric figures consisting of the 16 possible combinations of two values on each of the following four dimensions: (a) color (red-green), (b) size (1-2 in.), (c) number (1-2), and (d) form (square-triangle). The stimulus cards were randomized, with the restriction that no response category would appear more than four times consecutively.

\section{DESIGN}

The experimental design was a 2 by 2 by 2 factorial, with 10 Ss per cell. The factors in this design were: (a) instructions, (b) rule, and (c) percentage of positive and negative instances.

Two sets of instructions were employed. Half the Ss were given the following instructions: "This is a concept-learning experiment. Your task is to learn the concept. Same of the cards I show you will be examples of the concept, and some will not be examples of the concept." The remaining Ss were told: "This is a learning experiment. Some of the cards I show you will be 'yeses,' and some of the cards will be 'nos.' Your task is to learn which cards are 'yeses,' and which cards are "nos." "

The Ss were required to solve one of the two rules (conjunctive or disjunctive), under either a naturally occurring percentage of positive and negative instances (one-quarter positive and three-quarters negative for the conjunctive rule and the reverse for the disjunctive rule) or an equal percentage of positive and negative instances. For both the conjunctive and disjunctive rule, "red" and "square" were the relevant attributes.

\section{PROCEDURE}

Each $S$ was brought individually into the experimental room and seated across the table from $E$. One of the two sets of instructions was given, and $S$ was then presented with the stimulus cards, one at a time. S was asked to classify each card either as a "yes" or "no," or as an "example". or "not an example" of the concept, depending upon the instruction condition. After each classification, $S$ was given verbal feedback from E ("right" or "wrong"), and if $S$ was wrong, E gave him the correct classification for that particular card. This procedure continued until $S$ reached a criterion of 16 consecutive correct responses.

\section{RESULTS}

Since the variances of the eight experimental groups were not homogeneous, all analyses were performed on logarithmic transformations of the data. Table 1 presents the geometric means for both trials and errors to criterion.

Since, in most cases, there was no difference in the results of the analyses of trials and errors, only the results of the errors will be reported, except in those cases in which the results for the trials and errors differed.

A 2 by 2 by 2 analysis of variance of errors showed the instructions $(F=25.81$, $\mathrm{df}=1,72, \mathrm{p}<.01)$, rule $\quad(\mathrm{F}=33.18$, $\mathrm{df}=1,72, \mathrm{p}<.01)$, and percentage of positive and negative instances $(F=69.27$, $\mathrm{df}=1,72, p<.01$ ) effects to be significant sources of variance. The conjunctive rule, concept-learning instructions, and an equal percentage of positive and negative instances all led to better performance. The analysis showed a significant three-way interaction $(F=4.87, \mathrm{df}=1,72, \mathrm{p}<.05)$ among the three conditions, although in the analysis of trials to criterion, this interaction was not significant $(\mathrm{F}=1.50, \mathrm{df}=1,72)$. The significant three-way interaction was due to a lack of difference between the conjunctive and disjunctive problems in the unequal, yes-no condition $(t=.41$, df $=18)$. In all other conditions, the conjunctive problem was solved significantly faster than the disjunctive problem

\section{DISCUSSION}

The results indicate that both instructions and percentage of positive and negative instances do have rather large effects on performance. The instructions results in the unequal percentage condition replicated those obtained in a separate experiment with children (Denney, 1969), which also employed an unequal percentage of positive 
Table 1

Geometric Means of Trials and Errors to Criterion

\begin{tabular}{llrrrrr} 
& & \multicolumn{2}{c}{ Yes-No Instructions } & & \multicolumn{2}{c}{ Concept Learning Instructions } \\
\cline { 3 - 4 } & & Trials & Errors & & Trials & Errors \\
\hline \multirow{2}{*}{ Conjunctive } & Equal & 17.71 & 4.35 & & 6.59 & 3.10 \\
& Unequal & 104.00 & 31.20 & & 25.40 & 7.83 \\
Disjunctive & Equal & 53.79 & 15.30 & & 22.44 & 7.74 \\
& Unequal & 112.60 & 34.20 & & 73.38 & 21.25 \\
\hline
\end{tabular}

and negative instances. In both studies, no difference was obtained between conjunctive and disjunctive problems under the yes-no instructions, while the disjunctive problem was significantly more difficult under the concept-learning instructions. Although the concept-learning instructions in the present study were somewhat different from those used with the children, they did have the same effect on the relative difficulty of conjunctive and disjunctive problems. When the stimulus list was made up of an equal percentage of positive and negative instances, the results were somewhat different: the conjunctive problem was solved faster in all cor titions. It is not clear just why the two stimulus lists should have different effects $o_{1}$ the relative difficulty of the two problems when yes-no instructions are employed. However, it does seem reasonable to conclude that, at least in some cases, instructions do affect the relative difficulty of conjunctive and disjunctive problems.

The effect of concept learning instructions was to facilitate performance relative to yes-no instructions in all cases. One possible explanation for this facilitation may be that concept-learning instructions set the $S$ to look for some consistency within the positive class, while the yes-no instructions may have encouraged rote learning of all the stimulus patterns in both the positive and negative classes. Paying attention to just one class rather than two would reduce the amount of information to process, and should thus lead to faster solution. This explanation, however, does not really appear adequate. If Ss with the yes-no instructions were memorizing the stimuli in both classes, we would not expect a difference between conjunctive and disjunctive problems, since the problems are logically equivalent. On the other hand, if concept learning instructions focus the $S$ on the positive class, the conjunctive problem should take fewer trials, since the positive class on the conjunctive problem contains less varied information than the positive class on the disjunctive problem. Thus, an interaction between rule and instructions would be expected. This interaction, however, was not obtained. The expected lack of difference between the two problems under yes-no instructions was obtained only with the stimulus list composed of an unequal percentage of positive and negative instances. Further research is needed to determine why percentage of positive and negative instances should affect the relative difficulty of conjunctive and disjunctive problems when yes-no instructions are employed.

An equal percentage of positive and negative instances also led to faster solution in all conditions than an unequal percentage. This finding is relevant to the question of what type of instance $S$ is better able to use. Early research, investigating the learning of conjunctive concepts, demonstrated that Ss were better at using positive information than negative information (Smoke, 1933; Hovland \& Weiss, 1953). More recent evidence (Bourne \& Guy, 1968) indicates that the type of instance $S$ is better able to use depends on the rule $S$ is working with. If Ss use more of the information available in positive instances than in negative instances, the conjunctive problem should be learned faster when the positive and negative instances are equalized because of the larger concentration of positive instances that are most informative to $S$. On the other hand, the disjunctive problem should take longer to solve when the percentages of positive and negative instances are equalized, because the proportion of the most informative type of instance would be decreased. In fact, however, equalizing the percentage of positive and negative instances facilitated performance on both conjunctive and disjunctive problems. These results are in agreement with the results that Bourne \& Guy (1968) obtained with attribute-identification tasks. These authors compared conjunctive, disjunctive, and c onditiona 1 rules under attribute-identification and rule-learning procedures. Their training stimulus lists consisted of either all positive or all negative, or of a mixture of positive and negative, instances. The attribute-identification results indicated that Ss generally performed better on test trials containing both positive and negative instances when trained with instances from the smaller, more homogeneous class. The opposite was obtained for the rule-learning procedure. In the present study, the complete learning situation was similar to the attribute identification situation in the Boume and Guy study, in that the Ss learned more rapidly when presented with more instances of the smaller, more homogeneous, class. In the present study, Ss may have learned the conjunctive problem by focusing on the positive class, and the disjunctive problem by focusing on the negative class; thus, increasing the concentration of the type of instance $S$ focuses upon in the equal percentage condition gave $S$ more usable information within a given number of trials and, consequently, led to solution in a fewer number of trials. Disjunctive problems may take longer to solve, because, as Conant \& Trabasso (1964) have suggested, Ss learn to focus on the positive class more rapidly than they learn to focus on the negative class.

\section{REFERENCES}

BOURNE, L. E., JR., \& GUY, D. E. Learning of conceptual rules. II. The role of positive and negative instances. Journal of Experimental Psychology, 1968, 77, 488-494.

CONANT, M. B., \& TRABASSO, T. Conjunctive and disjunctive concept formation under equal-information conditions. Journal of Experimental Psychology, 1964, 67, 250-255.

DENNEY, N. W. The effects of varying instructions on conjunctive and disjunctive learning in children. Paper presented at the biennial meeting of the Society for Research in Child Development, Santa Monica, California, March 1969.

HOVLAND, C. I. \& WEISS, W. Transmission of information concerning concepts through positive and negative instances. Journal of Experimental Psychology, 1953, 45, 165-182.

KING, W. L A developmental study of rule learning. Journal of Experimental Child Psychology, 1966, 4, 217-221.

SMOKE, K. L. Negative instances in concept formation. Journal of Experimental Psychology, 1933, 16,583-588.

\section{NOTE}

1. This research was supported by Public Health Service Predoctoral Fellowship 7-F 1-MG-34,313-01A1 from the National Institute of Mental Health. The author wishes to express her appreciation to Dr. Joseph C. Campione for his assistance with the study and to Scott Barclay for his help in programming the data analysis. The authors' address is: Developmental Psychology Laboratory, University of Washington, Seattle, Washington 98105. 